\title{
Food, GI-tract Functionality and Human Health Cluster: PROEUHEALTH
}

\author{
T. Mattila-Sandholm, M. Blaut, C. Daly, L. De Vuyst, J. Doré, G. Gibson, H. Goossens, \\ D. Knorr, J. Lucas, L. Lähteenmaki, A. Mercenier, M. Saarela, F. Shanahan and \\ W. M. de Vos
}

Correspondence to: Prof Tiina Mattila-Sandholm, VTT Biotechnology, PO Box 1500, FI-02044 VTT, Finland. Tel: +3589456 5200; Fax: + 3589455 2103; E-mail: tiina.mattila-sandholm@vtt.fi

Microbial Ecology in Health and Disease 2002; 14: 65-74

The Food, GI-tract Functionality and Human Health (PROEUHEALTH) Cluster brings together eight complementary, multicentre interdisciplinary research projects. All have the common aim of improving the health and quality of life of European comsumers. The collaboration involves 64 different research groups from 16 different European countries and is coordinated by leading scientists. The research results from the cluster are disseminated through annual workshops and through the activities of three different platforms: a science, and industry and a consumer platform. The cluster started in 2001 and will end in 2005.

\section{INTRODUCTION}

Probiotics, prebiotics, and synbiotics aimed at improving intestinal health currently represent the largest segment of the functional foods market in Europe, Japan and Australia. Evidence continues to emerge demonstrating that these ingredients have a potential to improve human health in specific intestinal disorders. The European Commission, through its $5^{\text {th }}$ Framework Programme, is presently investing a substantial research effort in the intestinal microbiota, the interaction with its host, and methods to manipulate its composition and activity for the improvement of human health. Eight multicentre interdisciplinary research projects currently cover a variety of research topics believed to be crucial for the development of efficacious probiotic foods, ranging from the understanding of probiotic mechanisms at a molecular level to developing technologies that ensure delivery of stable products and demonstrating safety and efficacy in specific disorders. This concerted research effort promises to provide an appreciable understanding of the human intestinal microbiota's role in health and disease, and new approaches and products to tackle a variety of intestinal afflictions.

A number of steps are essential in the development of efficacious probiotic and prebiotic functional foods (Fig. 1). A prerequisite for mechanistic studies of probiotic action is an understanding of the composition and activity of the intestinal microbiota as well as interactions with the host in both healthy and diseased individuals. High throughput molecular methods are required to examine the intestinal microbiota and to track the location and activity of probiotic strains in the intestinal tract. An understanding of the mechanisms by which probiotics exert beneficial effects on human health allows selection of strains with appropriate traits for hypothesis-driven clinical studies. The safety of new strains must be demonstrated before they are used in human clinical trials. An important area of research is the establishment of technologies maximizing the stability of functional traits of probiotics during manufacture, formulation, storage and in the intestinal tract. Additionally, the efficacy of products may be enhanced by exploiting synergistic interactions between functional ingredients as is potentially the case with synbiotics. Finally, an understanding of the most appropriate methods to communicate the benefits of the functional foods to consumers and the influence of health messages on consumer choice is essential to ensure that products are appropriately applied and targeted to benefit specific populations.

The Food, GI-tract Functionality and Human Health (PROEUHEALTH) Cluster brings together eight complementary, multicentre interdisciplinary research projects (Fig. 2). Their common aim is to improve the health and quality of life of Europeans. The collaboration involves 64 different research groups from 16 different European countries and is coordinated by leading European scientists (see Appendix). The research results from the cluster will be disseminated through various publications, annual workshops and through the activities of three different platforms: a science, an industry and a consumer platform (Fig. 3). The cluster started in 2001 and will end in 2005. 
- Which bacterium is which?

- Second generation probiotics

- New therapeutics with potential against IBD

- A healthier retirement

- Keeping probiotics alive and healthy so that they can keep you healthy

- Probiotics can defend against bad bacteria

- Ensuring the safety of probiotic bacteria

- Why are probiotics effective

Fig. 1. The cluster projects in consumers language.

\section{DESCRIPTION OF PROJECTS}

\section{Developing research tools}

Project 1: Development and application of high throughput molecular methods for studying the human gut microbiota in relation to diet and health-MICROBE DIAGNOSTICS

The microbial gut ecosystem has a major impact on the health and well-being of humans. Since the make-up and activity of the gut microorganisms, collectively known as the microbiota, are highly influenced by diet, the project is designed to provide a better understanding of its impact on human health and easy-to-use methods for monitoring its composition. Novel methods are being developed to understand and exploit the nutrition-driven impact of the human gut microbiota on health. Since the presently used methods for analysing the intestinal microbiota are time consuming and tedious, high throughput methods for the automated detection of fluorescently labelled cells based on microscopic image analysis, flow cytometry and DNA arrays will be developed. In situ detection methods for monitoring bacterial gene expression at the cellular level will be developed to monitor the impact of dietary constituents on the transcription of bacterial genes. Samples from human populations will be analyzed with the developed methods to identify important factors of microbiota development, composition and activity.

Objectives. The project is aimed at developing, refining, validating and automating the most advanced molecular methods for monitoring the composition of the gut microbiota and bacterial gene expression in selected human populations in response to diet and life style. The specific objectives of the project are (1) to improve and facilitate gut microbiota monitoring with molecular methods, (2) to understand antagonistic and synergistic interactions of the intestinal microbiota in response to nutrition, and (3) to find links between major dysfunctions and the intestinal microbiota.

Future results and achievements. The project is expected to provide methods that allow rapid detection of intestinal bacteria and their activities. The application of the developed methods will provide baseline data on the intestinal microbiota composition in response to origin and lifestyle. The project will also provide fundamental information on the biodiversity and phylogeny of the human gut microbiota. The obtained data will be used to develop a mechanistic concept for diet-induced microbiota development and to define the role of the intestinal microbiota in disease development. The project thereby contributes to investigate the role and impact of food on physiological function, the development of foods with particular benefits, and links between diet and chronic diseases.

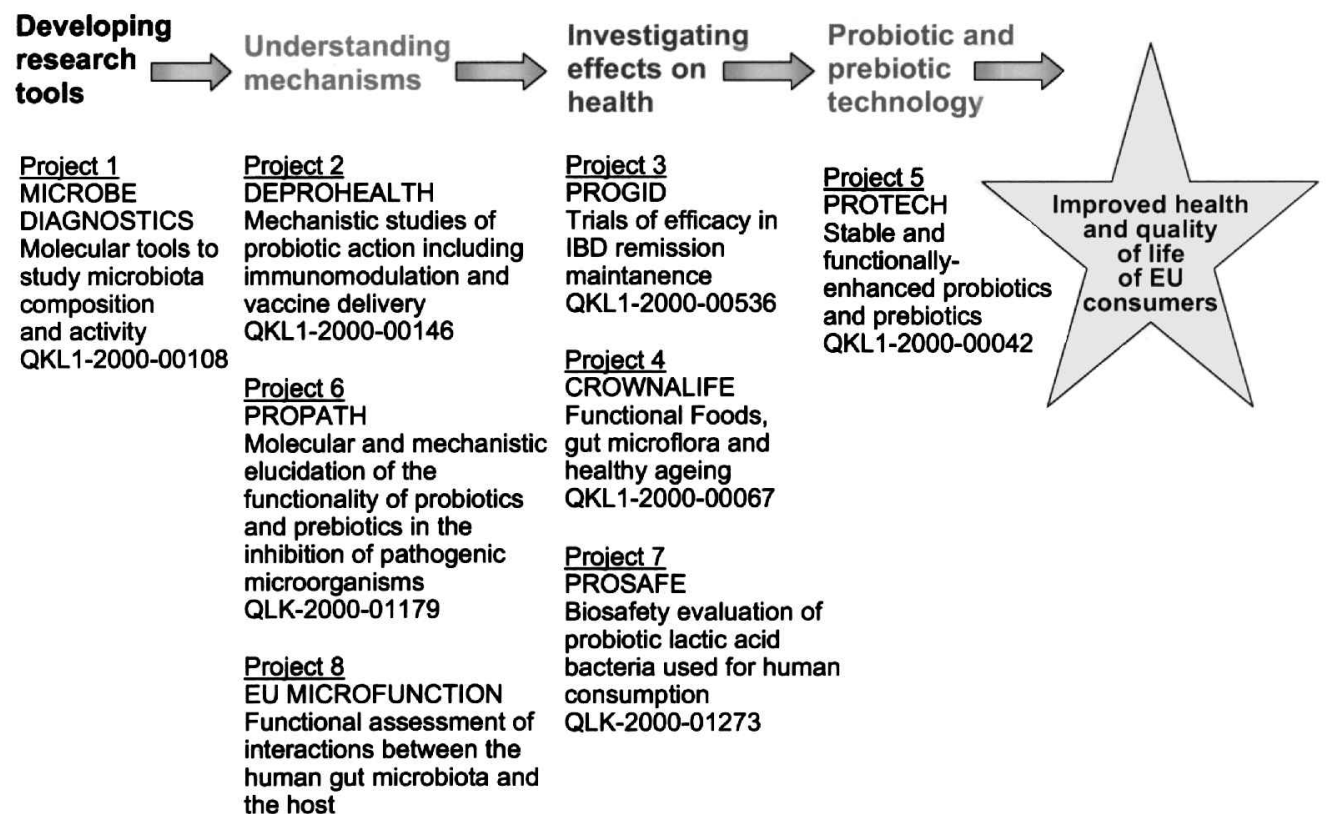

Fig. 2. Food, GI-tract functionality and human health cluster. 


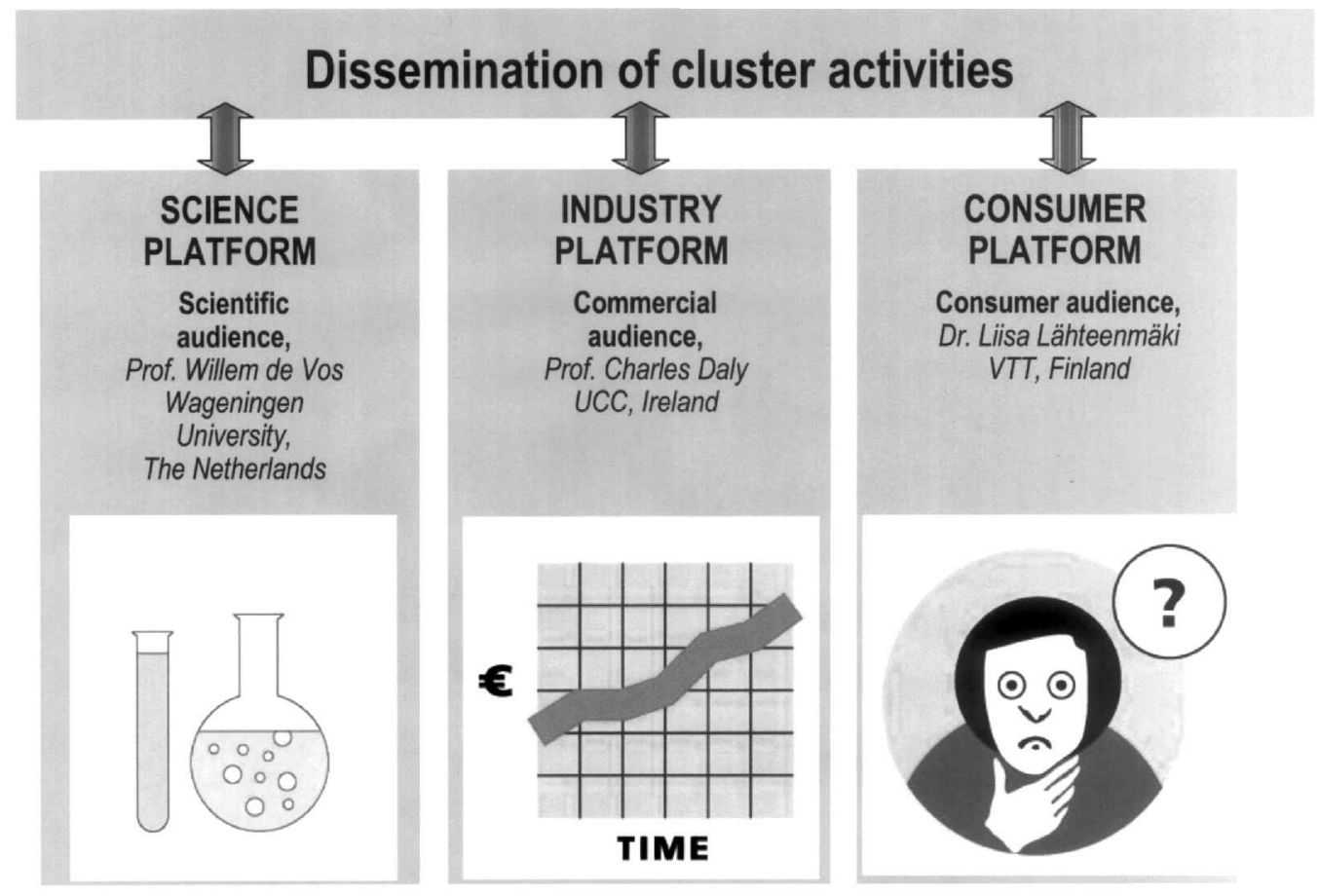

Fig. 3. Three platforms will disseminate the aims and findings of the cluster to targeted audiences.

The project will provide technological solutions for the rapid detection of gut microorganisms with a big potential for a wide range of applications. The screening of gut microbiota composition will be applied in relevant biomedical research areas. It will facilitate the diagnosis of a disturbed microbiota and the development of rational therapies based on our knowledge of disease mechanisms. It will support the development of functional foods aimed at improved gastrointestinal function and other health benefits.

\section{Understanding mechanisms}

\section{Project 2: Probiotic strains with designed health properties- DEPROHEALTH}

Lactic acid bacteria (LAB) are well-known for their extensive use in the preparation of fermented food products. In addition, the potential health benefits they may exert in humans have been intensively investigated during the last century. However, the mechanisms underlying the healthpromoting traits attributed to $\mathrm{LAB}$, especially lactobacilli, remain vastly unknown and this has impaired the rational design of probiotic screening methods with accurate predictive value. This project aims at establishing a correlation between in vitro tests and mouse models mimicking important human intestinal disorders such as inflammatory bowel disease, Helicobacter pylori and rotavirus infections. As these diseases correspond to major public health problems, a second generation of probiotic strains with enhanced prophylactic or therapeutic properties will also be designed during the project. These designed strains and the isogenic parental ones will be used to unravel mechanisms involved in the immunomodulation capacity of specific Lactobacillus strains.

Objectives. The general aim of this project is to acquire knowledge about the molecular factors affecting the immunomodulation and/or immunogenicity of selected probiotic lactobacilli to allow for developing isolates with enhanced protective or therapeutic effect. The objectives of the proposal are, on the one hand, to unravel mechanisms and identify key components of the immunomodulation capacity of probiotic lactobacilli, and on the other, to design a so-called second generation of probiotic strains with enhanced properties against gastro-intestinal disorders.

Two types of gastro-intestinal diseases are targeted: inflammations such as inflammatory bowel disease (IBD) and infections such as those caused by $H$. pylori and rotavirus. For each of them, atherapeutic or prophylactic (i.e. vaccine) recombinant probiotic strains will be constructed and tested in relevant animal models (i.e. mimicking the human disease) to evaluate their capacity to induce or modulate the immune response in the proper way. This data will be correlated to in vitro testing of the immunomodulation capacityin an attempt to identify/develop screening methods that, in the future, will allow isolation of difficient probiotic strains targeted at specific applications. Recombinant DNA technology will also be used to assess the importance of specific bacterial cell wall components and adhesion factors in immunomodulation. 
Future results and achievements. Two types of modified probiotic strains will be constructed: mutant strains affected in their cell wall composition and adhesion proteins as well as recombinant strains with enhanced therapeutic or protective properties, focusing on gastrointestinal diseases of inflammatory or infectious origin. The final goal would be to prove that designed probiotic strains could be used as original therapeutic agents as, if successful, they would lead to novel anti-inflammatory treatments or oral vaccines against Helicobacter pylori and rotavirus. It is also expected that, by the end of the study, major progress will have been achieved towards the rational design of simplified probiotic screening methods with accurate predictive value.

\section{Project 3: Molecular analysis and mechanistic elucidation of the functionality of probiotics and prebiotics in the inhibition of pathogenic microorganisms to combat gastrointestinal disorders and to improve human health-PROPATH}

Recently, much attention has been paid to the health-promoting properties of lactobacilli and bifidobacteria. Probiotics and prebiotics are the driving forces of the functional foods market. However, a major problem is that many of these health-promoting properties are still questioned. For instance, the fundamental basis of the inhibition of Gramnegative pathogenic bacteria, such as the enterovirulent diarrheagenic Salmonellae, and Helicobacter pylori, causing gastritis and gastric ulcer disease, by probiotic LAB has not been elucidated. This project will focus on the identification of the responsible compounds. In addition, the mechanism of the inhibition of Gram-negative pathogens by probiotic lactobacilli and bifidobacteria will be studied using coculture models (simulated gut fermentations, human cell lines and animal models).

Objectives. The aim is to obtain a selection of probiotic lactobacilli and bifidobacteria that display a clear inhibition of diarrheagenic Gram-negative pathogenic bacteria and Helicobacter pylori. Thereafter the project focuses on identifying the metabolite(s) that are responsible for the inhibition and/or killing of Gram-negative pathogenic bacteria at the same time as they have the conditions and kinetics of the production of antimicrobials active towards Gram-negative pathogens and to predict their in vivo action. One of the aims will be to establish co-culture models (simulated gut fermentations, human cell lines, animal models) and to study the interaction between inhibitory strains of LAB and Gram-negative pathogens causing gastrointestinal disorders. Finally the selected probiotic LAB will be tested in clinical studies.

Future results and achievements. The results of the molecular analysis and mechanistic elucidation of the functionality of probiotics and prebiotics in the inhibition of pathogenic microorganisms will result in (1) a project culture collection of probiotic strains of lactobacilli and bifidobacteria together with data on their characteristic and inhibitory spectrum; (2) a molecular typing method for selected probiotic strains; (3) identified compounds responsible for the inhibition of Gram-negative pathogenic bacteria; (4) conditions of antimicrobial production in the gut environment and (5) co-culture models (simulated gut fermentations, human cell line cultures, animal models) showing the inhibitory action by the probiotic strains.

\section{Project 4: Functional assessment of interactions between the human gut microbiota and the host-EU \& MICROFUNCTION}

The aim of this project is to identify the effects of dietary modulation on the human gastrointestinal microbiota. The influence of live microbial food supplements (probiotics), dietary carbohydrates known to have a selective metabolism (prebiotics), and a combination of these two approaches (synbiotics) will be ascertained. The main objectives are to clarify effects on the normal gut microbiota, and on host gastrointestinal function, as well as determine mechanisms involved in pro-, pre- and synbiotic functionality. These objectives will be achieved through exploitation of model systems and state-of-the-art technology.

Objectives. The principal objectives are to determine the efficacy and safety of probiotics and prebiotics, determine effective doses/combinations, identify mechanisms of action and investigate impacts on host function. An important aspect will be the development of new synbiotics and their use in a human trial. The work planned aims to identify the mechanisms of effect and to produce valuable information on the influence of dietary intervention on the activities of human gut microbiota. In addition, it will provide essential means of validating probiotics and prebiotics and give information on the optimal combinational approach.

There is currently an imperative requirement to identify the realistic health outcomes associated with probiotic and prebiotic intake and, importantly, give rigorous attention towards determining their mechanisms of effect. This project will aim to do so through investigating probiotic and prebiotic influence on host functionality, including microbiological and physiological aspects.

Future results and achievements. The aim is to identify mechanistic interrelationships through fundamental scientific approaches. The following milestones will be achieved: efficient prebiotics and required dosage; active synbiotics; effects of functional foods on bacterial translocation; effects on host gene interactions; safety of functional foods determined; effects on selected health indices in humans.

\section{Investigating effects on health}

\section{Project 5: Probiotics and gastrointestinal disorders: Con- trolled trials of European Union patients-PROGID}

This project will assess the efficacy of two previously selected probiotic microorganisms, administered as dried fermented 
milk products, in alleviating the effects of inflammatory bowel disease (IBD) - Crohn's disease and ulcerative colitis. Specifically, two distinct long-term (12 month), large-scale, multi-center, randomized, double blind, placebo-controlled feeding trials will be performed within a subset of the European Union population suffering from these gastrointestinal disorders.

Background and objectives. In recent years, the International Organization for the Study of Inflammatory Bowel Disease and others have published prominent articles highlighting the necessity for standards in the design of trials evaluating therapies in gastrointestinal disorders. In the assessment of probiotic products, however, most studies have been small, uncontrolled and poorly documented with imprecise definition of the endpoints. In response, the European Commission has funded the PROGID project which will evaluate two specific probiotic microorganisms in patients with ulcerative colitis or Crohn's disease from diverse geographical locations. The participating centers in these studies will assess the efficacy of Bifidobacterium infantis UCC35624 and Lactobacillus salivarius UCC118 in one year, randomized, double blind, placebo-controlled trials for maintenance of remission. Both of the selected probiotic strains have a history of safety and efficacy in healthy adults and relapsed inflammatory bowel disease patients.

Future results and achievements. The anticipated results from the PROGID project include: the provision of qualitative and quantitative evidence that the evaluated probiotics may (or may not) have a role in maintaining remission of Inflammatory Bowel Disease (IBD); confirmation, or otherwise, of the involvement of specific members of the gastrointestinal microbial flora populations as causative or contributory agents of inflammatory bowel disease; generation of linear physiological and immunological data relevant to the disease and remission states of inflammatory bowel disease; creation of a greater awareness among the EU population of functional foods and their potential benefits in maintaining healthy lifestyles; and the establishment of a repository of biological samples obtained from across the EU.

\section{Project 6: Functional food, gut microflora and healthy ageing-CROWNALIFE}

Elderly people represent an increasing fraction of the European population. Their higher susceptibility to degenerative and infectious diseases lead to rising public health and social concerns. Appropriate preventive nutrition strategies can be applied to restore and maintain a balanced intestinal microbiota exerting protective functions against the above disorders. The project is based on the application of selected biomarkers in hypothesis-driven human studies. We will identify the structural and functional specificity of the elderly's intestinal microbiota across Europe. Using this baseline information, we will investigate functional food-based preventive nutrition strategies aiming to beneficially affect the functional balance of the elderly's intestinal microbiota. Expected outcomes include nutritional recommendations as well as new concepts and prototype functional foods, specifically adapted for health benefits to the elderly population.

Objectives. The overall objective is to improve the quality of life of the elderly throughout the third age, with emphasis on the preservation of the period of independence recognized as the crown of life. The focus is on preventive nutrition and the application of functional food to derive health benefits for the ever-increasing elderly European population. Based on hypothesis-driven human studies, the specific objectives of the project are (1) to assess structural and functional alterations of the intestinal microbiota with ageing and across Europe; and (2) to validate functional foods-based preventive nutrition strategies to restore and maintain a healthy intestinal microbiota in the elderly. Implementations include nutritional recommendations as well as new concepts and prototype functional food specifically adapted for health benefits to the elderly population.

Future results and achievements. The results will establish six steps: (1) the assessment of the gut microbiota diversity and composition in the European elderly population, and identification of its alterations with ageing (baseline human study); (2) the assessment of modulation of the intestinal microbiota (intervention human study with functional food), and potential health benefits towards degenerative pathologies and infectious diseases; (3) the improved health status of the ageing population via specific nutritional recommendations; (4) the nutritional guidelines based on the complete assessment of a synbiotic product; (5) the design and provision of adapted food supplements directed towards intestinal microbial function, and (6) the validation of processes and rationale for the design of a new generation of functional foods to satisfy health benefits for the elderly, based on innovative technologies.

\section{Project 7: Biosafety evaluation of probiotic lactic acid bac- teria used for human consumption-PROSAFE}

Safety assessment is an essential phase in the development of any new pro- or prebiotic functional foods. The safety record of probiotics is good, and lactobacilli and bifidobacteria have a long history of safe use. However, all probiotic strains must be evaluated for their safety before being used in human clinical studies and in functional food products. Conventional toxicology and safety evaluation alone is of limited value in the safety evaluation of probiotic bacteria. Instead, a multidisciplinary approach is necessary involving contributions from pathologists, geneticists, toxicologists, immunologists, gastroenterologists and microbiologists. 
Objectives. The safe use for human consumption of probiotic strains, selected LAB including lactococci, lactobacilli, pediococci and bifidobacteria, and other food-associated microorganisms such as enterococci will be assured by proposing criteria, standards, guidelines and regulations. Furthermore, procedures and standardized methodologies of pre-marketing biosafety testing and post-marketing surveillance will be provided. The specific objectives of the project will include five stages: (1) the taxonomic description of probiotics, LAB and other food-related microorganisms; (2) the detection of resistance and horizontal transfer of antibiotic resistance genes; (3) the careful analysis and definition of the nonpathogenic status of probiotic LAB; (4) the immunological adverse effects of the studied bacteria in the EAE model and (5) the survival, colonization, and genetic stability of probiotic strains in the human gut.

Future results and achievements. Future results will finalize important goals relating to probiotic strains, selected LAB and other food-associated microorganisms and consists of (1) establishing a culture collection and database; (2) providing standardized methodologies to detect antibiotic resistance; (3) investigating the (potential) virulence properties and their association with clinical disease and results obtained in rat endocarditis models; (4) studying potential immunological adverse effects; (5) analyzing the genetic stability and colonization of probiotic strains in the human gastro-intestinal tract, and (6) providing recommendations for biosafety evaluation of probiotic strains.

\section{Probiotic and prebiotic technologies}

Project 8: Nutritional enhancement of probiotics and prebiotics: Technology aspects on microbial viability, stability, functionality, and on prebiotic function-PROTECH

Maintaining the functional properties of probiotics during manufacture, formulation and storage, are essential steps in delivering health benefits to consumers from these products. The overall objective of this project is to address and overcome specific scientific and technological hurdles that impact on the performance of functional foods based on probiotic-prebiotic interactions. Such hurdles include the lack of a strong knowledge on the primary factors responsible for probiotic viability, stability, and performance. Limited information is available on the impact of processing, storage and food matrices or food constituents on probiotic viability, stability and functionality. Furthermore, sufficient data are missing about the interactions between probiotics and prebiotics in functional foods prior to consumption.

Objectives. This project has three general objectives: (1) to explore the effects of processing on the stability and functionality of probiotics and on the performance of prebiotics; (2) to apply selected processing techniques for prebiotic modification to identify and optimize probiotic- prebiotic combinations; and (3) to use the information generated as the basis for new process and product options.

As a result of achieving these objectives, selected prebiotics in combination with tailored manufacturing processes will not only contribute to probiotic functionality but also improve viability and stability of probiotic cultures within food matrices during processing and storage.

The project is divided in five specific objectives which are:

(1) Effects on probiotic viability: The aim is to consolidate quantitative data of processing effects on the physiology and viability of probiotic organisms. This will include effects during different fermentation processes, and freezing and drying strategies and also during passage through the GI-tract. Effects on cell composition including stress responses, cell membrane composition and leakage, and cell viability will be measured. A viability model for probiotic cultures will be developed and validated.

(2) Effects on probiotic stability: Experiments will be performed to investigate the influence of media composition and processing conditions on probiotic stability. This includes cryotolerance, resistance to dehydration, storage and genetic stability. Stability of the important probiotic characteristics of strains will also be investigated including acid and bile tolerance and adhesion to intestinal cells. New formulation techniques including micro-encapsulation will be assessed in real food matrices including dairy and cereal based foods.

(3) Probiotic-prebiotic interaction: The ultimate objective is to optimize probiotic-prebiotic interaction for maximum probiotic performance in culture, in food systems, and in the GI-tract. Special attention will be given to indigestible oligo- and polysaccharides with respect to fermentability and to production of short chain fatty acids in animal models and in vitro.

(4) Prebiotic function: New prebiotics will be developed that are tailor-made for the stabilization and optimal performance of probiotics. These will be examined for their physiological properties and their organoleptic, and physicochemical properties will be technologically evaluated in real foods.

(5) Probiotic functionality: The effect of growth conditions and stress environments on the functionality of probiotic bacteria will be examined. Stresses tested will include high acid, bile, temperature, oxygen and osmotic tension. In addition, high hydrostatic pressure and controlled permeabilization technologies will be evaluated stress preconditioning of LAB, including bifidobacteria. Cross protection against other stresses will be examined.

Future results and achievements. Expected achievements include the establishment of unique data sets that include the identification of critical process parameters for probiotics and prebiotics and results from systematic studies suggesting means to overcome existing process and 
product limitations. The compilation of protocols for probiotic performance, prebiotic function and probiotic-prebiotic interactions will also be provided. Further, it is expected to establish probiotic viability models and functionality biomarkers. In addition, it is attempted to achieve optimization of probiotic viability, stability in culture and real food systems at pilot plant scale, generation and modification of unique prebiotics, of probiotic interactions and of environmentally and processing induced functionality of probiotics. Application of the expected results will lead to new process concepts for probiotics, for prebiotics and for probiotic-prebiotic combinations. Special emphasis of the development of product concepts will be on cereal and dairy-based products and on the development and incorporation of unique plant based prebiotics for optimal interaction between prebiotics performance and probiotics function.

\section{Consumers and perceived health benefits of probiotics}

Science-based knowledge on how probiotic bacteria can promote our well-being has increased in recent years. The success of probiotic products will be determined by consumers' willingness to buy and eat them. The perceived benefits of these new products are key factors for consumer acceptance. Some of the benefits are relevant to a large group of people, but others benefit only well-defined target groups. For ordinary consumers, preventing traveller's diarrhea or helping with temporary gut disorders is a clear advantage, but still the motivation to use probiotics for patients suffering from irritable bowel syndrome or inflammatory bowel disease is likely to be higher if the probiotics can, at least partly, replace medication and reduce or even prevent symptoms.

Functional foods, i.e. products promising specified effects on physiological functions, represent a new kind of health message for the consumers. In traditional nutritional messages the emphasis is on diet and on avoiding or favouring certain types of foods rather than giving recommendations on particular products. Probiotic and other products containing the new kind of health message appear to better respond to the needs of different people than the idea of a nutritionally balanced diet.

Although the most enthusiastic users of functional foods are believed to be middle aged women who already follow healthy diets, in previous studies in Finland the general health interest, which measure people's willingness to comply with nutritional recommendations, has not been strongly linked with the willingness to use functional foods. The well-defined benefits promised by probiotic foods contain a positive message to consumers. The idea of gaining a benefit by using a product rather than avoiding a slow development of chronic diseases forms a different message. For Irish consumers the most interesting claims were related to heart diseases, cancer and bone health. Surprisingly, probiotics were not well recognized, even though yoghurt was among the most frequently used functional foods.

Developing probiotic foods also introduces the novelty aspect into the product. In general, consumers tend to be suspicious towards novelty in foods because it means uncertainty and thus threat. Therefore, the benefits promised by these new food products will be weighed against the perception of possible risks. Highly developed technologies are often involved in manufacturing the new probiotic foods. In several European countries healthiness has been associated with naturalness. The need for highly advanced technology in the production of probiotic foods may lower the perceived naturalness, which may create distrust towards functional foods among consumers. Consumers seem to find functions that enforce the natural properties of a product more acceptable than those functions that are artificially added and in disaccordance with the earlier image of the product. Therefore, in addition to technological considerations, adding probiotics into food products require understanding of the existing beliefs consumers have about these products.

Consumer responses to functional foods and their health-related messages require more research. Communicating the gut health messages effectively to consumers with varying age and cultural background remains one of the key issues. Besides this we need tremendous efforts towards communication and cooperation between medical doctors, nutritionists, pharmaceutics. As the information is a vital factor, trust between all sides is required, so that messages will be given attention. The differences between functional properties of various strains are difficult to explain to consumers and tools for doing this should be created. For some consumers, food products working almost like medicines may be hard to approve of. Furthermore, food products need to have a place in the daily food system, whereas medicines are taken upon instructions, and using food as a replacement for medicines requires adjustments in eating habits as well. Probiotic products can improve human health only if they are eaten frequently. To ensure this the above issues must be addressed so that an appropriate set of products which will be accepted and consumed can be developed.

\section{CONCLUSIONS}

Health is one of the main reasons behind food choices, but still other factors are taken into consideration by the consumer. As pleasantness and sensory quality can be experienced directly, they are known to be the essential factors in repeated choices. Even with foods aimed for health, the taste has to be good for securing repeated choices. Probiotics modifying the gut microbiota have physiological effects that cannot be experienced directly. The credibility of the health-related messages is therefore the critical goal in aim to guarantee that the product has a reward value for the consumer. Consumers are selective in 
their attention so that messages that are relevant to us and which are congruent with our earlier beliefs are easily accepted. Therefore, food-related messages have to correspond with our existing beliefs and motivation to use a product. Preventing a possible stomach upset has little relevance to a healthy person, but can make a vast difference for someone suffering from IBD or someone who easily catches traveller's diarrhea.

Keeping this in mind the microbes with health impact are and will remain an important functional ingredient for years to come. New strains will be identified and foods will be developed to fulfill the needs of specific consumer groups. Increased understanding of interactions between the gut microbiota, diet and the host will open up possibilities of producing novel ingredients for nutritionally optimized foods promoting consumer health through microbial activities in the gut.

The future research on food and microbes with health impact will continue to develop specifically aimed at (1) exploring the mechanisms of action of microbes and their health effects in the GI-tract, especially in healthy individuals; (2) developing sophisticated diagnostic tools for the gut microbiota and biomarkers for assessing their functionality; (3) examining the effects of food-derived bioactive compounds on GI-diseases, GI-infections, and allergies; (4) developing new therapeutic and prophylactic treatments for different patient and population groups; (5) realizing molecular understanding of immune modulation by bacteria with health-effects; (6) elucidating the role of colon microbiota in the conversion of bioactive compounds; (7) analyzing the effects of the metabolites to the colon epithelium or the effects after absorption; (8) ensuring the stability of microbes with health effects and their bioactive compounds also in new types of food applications by developing feasible technologies; and finally (9) providing the safety of the functional ingredients.

The acceptance of probiotic, prebiotic, symbiotic products in the future will depend on the solid proof of the health benefits they promise at the moment. Therefore, research that provides the scientifically sound evidence to back up the health claims is needed. How to make the knowledge produced by science comprehensible to the consumer is the major challenge, as the laymen thinking is based on approximation and black-and-white views whereas scientific thinking deals with probabilities and degrees of uncertainty. Consumers' trust in the information depends on the source and content of message. The critical point for the producers of probiotic foods is to gradually build and ensure consumer trust.

\section{ACKNOWLEDGEMENT}

Financial support from the European $5^{\text {th }}$ Framework programme: Quality of Life and Management of Living Resources, Key action 1.

\section{SELECTED REFERENCES}

Alander M, Mättö J, Kneifel W, et al. Effect of galacto-oligosaccharide supplementation on human faecal microflora and on survival and persistence of Bifidobacterium lactis $\mathrm{Bb}-12$ in the gastrointestinal tract. Int Dairy J 2001; 11: 817-25.

Avonts L, De Vuyst L. Antimicrobial potential of probiotic lactic acid bacteria. Mededelingen van de Faculteit Landbouwkundige en Toegepaste Biologische Wetenschappen Universiteit Gent 2001; 66/3b: 543-50.

Bech-Larsen T, Grunert KG, Poulsen JB. The acceptance of functional foods in Denmark, Finland and the United States. MAPPWorking paper no 73, The Aarhus School of Business, 2001; 73.

Bengmark S. Ecological control of the gastrointestinal tract. The role of probiotic flora. Gut 1998; 42: 2-7.

Bogue J, Ryan M. Market-oriented New Product Development: Functional Foods and the Irish Consumer. Agribusiness Discussion paper No. 27, Department of Food Economics, University College, Cork, Ireland 2000; 27.

Collins, MD, Gibson, GR. Probiotics, prebiotics and synbiotics: dietary approaches for the modulation of microbial ecology. Am J Clin Nutr 1999; 69:1052-7.

Coconnier MF, Liévin V, Hemery E, Laboisse CL, Servin AL. Antagonistic activity of the human Lactobacillus acidophilus strain LB against Helicobater infection in vitro and in vivo. Appl Environ Microbiol 1998; 64:4573-80.

Coconnier MH, Liévin V, Lorrot M, Servin AL. Antagonistic activity of Lactobacillus acidophilus LB against intracellular Salmonella enterica Serovar Typhimurium infecting human enterocyte-like Caco-2/TC-7 cells. Appl Environ Microbiol 2000; 66: $1152-7$.

Crittenden R, Saarela M, Ouwehand A, et al. T. Lactobacillus paracasei $\mathrm{F} 19$ : survival, ecology and safety in the human intestinal tract. Microb Ecol Health Dis 2002; 3: 22-6.

Crittenden R, Laitila A, Forssell P, et al. Adhesion of bifidobacteria to granular starch and implications in probiotic technologies. Appl Environm Microbiol 2001; 67: 3469-75.

Devos WM. Advances in genomics for microbial food fermentations and safety. Current Opin Biotechnol 2001; 12: 493-8.

Dunne C, Shanahan F. The role of probiotics in the treatment of intestinal infections and inflammation. Curr Opin Gastroenterol 2002; 18: 40-5.

Dunne C. Adaptation of bacteria to the intestinal niche-probiotics and gut disorder. Inflammatory Bowel Diseases 2001; 7 : $136-45$.

Grangette C, Müller-Alouf H, Goudercourt D, Geoffroy M-C, Turneer M, Mercenier A. Mucosal immune responses and protection against tetanus toxin after intranasal immunization with recombinant Lactobacillus plantarum. Infect Immun 2001; 69:1547-53.

Grunert K, Bech-Larsen T, Bredahl L. Three issues in consumer quality perception and acceptance of dairy products. Int Dairy J 2000; 10: 575-84.

Heilig HG, Zoetendal EG, Vaughan EE, Marteau P, Akkermans AD, de Vos WM. Molecular diversity of Lactobacillus spp and other lactic acid bacteria in the human intestine as determined by specific amplification of $16 \mathrm{~S}$ ribosomal DNA. Appl Environm Microbiol 2002; 68: 114-23.

Kruse HP, Kleessen B, Blaut M. Effects of insulin on faecal bifidobacteria in human subjects. Br J Nutr 1999; 82: 37582.

Lappalainen R, Kearney J, Gibney M. A Pan-European survey of consumers attitudes to food, nutrition and health: an overview. Food Quality and Preference 1998; 9: 467-78. 
Liévin V, Peiffer I, Hudault S, Rochat F, Brassart D, Neeser J-R, Servin AL. Bifidobacterium strains from resident infant human gastrointestinal microflora exert antimicrobial activity. Gut 2000; 47: $646-52$.

Lähteenmäki L, Arvola A. Food neophobia and variety seekingconsumer fear or demand for new food products. In: Frewer LJ, Risvik E, Schifferstein H, eds. Food, people and society. A European perspective of consumer food choices. Berlin: SpringerVerlag, 2001: 161-75.

Mattila-Sandholm T, Blum S, Collins JK, et al. Probiotics: towards demonstrating efficacy. Trends Food Sci Technol 2000; 10: $393-9$.

Mattila-Sandholm T, Saarela M. Probiotic functional foods. In: Gibson GR, Williams CMF, eds. Functional foods-concept to product. Cambridge: Woodhead Publishing Limited, 2000: 287313.

Mattila-Sandholm T, Myllärinen P, Crittenden R, Mogensen G, Fonden R, Saarela M. Technological challenges for future probiotic foods. Int Dairy J 2001; 12: 1-10.

Marteau P, Pochart P, Doré J, Maillet C, Bernalier A, Corthier G. Comparative study of the human cecal and fecal flora. Appl Environ Microbiol 2001; 67: 4939-42.

Mercenier A, Mattila-Sandholm T. The food, GI-tract functionality and human health European research cluster, PROEUHEALTH. Nutr Metab Cardiovasc Dis 2001; 11: 1-5.

Naidu AS, Bidlack WR, Clemens RA. Probiotic spectra of lactic acid bacteria (LAB). Crit Rev Food Sci Nutrit 1999; 38:13-126.

Poulsen JB. Danish consumers' attitudes towards functional foods. MAPP Working paper no 62, The Aarhus School of Business, 1999:62.

Roininen K, Lähteenmäki L, Tuorila H. Quantification of consumer attitudes to health and hedonic characteristics of foods. Appetite 1999; 33: 71-88.

Saarela M, Mogensen G, Fonden G, Mättö J, Mattila-Sandholm T. Probiotic bacteria: safety, functional and technological properties. J Biotechnol 2000; 84: 197-215.

Salminen S, Bouley C, Boutron-Ruault, MC, et al. Gastrointestinal physiology and function - targets for functional food development. Br J Nutrit 1998; 80 (Suppl): 147-71.

Salminen S, Ouwehand AC, Isolauri E. Clinical applications of probiotic bacteria. Int Dairy J 1998; 8: 563-72.

Salminen S, von Wright A. Current probiotics-safety assured? Microb Ecol Health Dis 1998; 10: 68-77.

Salminen S, Isolauri E, Onnela T. Gut flora in normal and disordered states. Chemotherapy 1995; 41:5-15.
Salminen S, von Wright A, Morelli L, et al, Demonstration of safety of probiotics-a review. Int J Food Microbiol 1998; 44: 93-106.

Sanders ME, Huis in't Veld JHJ. Bringing a probiotic-containing functional food to the market: microbiological, product, regulatory and labeling issues. Antonie van Leeuwenhoek 1999; 76: $293-315$.

Satokari RM, Vaughan EE, Akkermans AD, Saarela M, De Vos WM. Polymerase chain reaction and denaturing gradient gel electroforesis monitoring of fecal bifidobacterium populations in a prebiotic and probiotic feeding trial. Syst Appl Microbiol 2001; 24: $227-31$

Schwiertz A, Le Blay G, Blaut M. Quantification of different Eubacterium spp in human fecal samples with species-specific $16 \mathrm{~S}$ rRNA-targeted oligonucleotide probes. Appl Environ Microbiol 2000; 66: 375-82.

Sghir G, Gramet A, Suau V, Rochet P, Pochart P, Doré J. Quantification of bacterial groups within human fecal flora by oligonucleotide probe hybridization. Appl Environ Microbiol 2000; 66: 2263-6.

Shanahan F. Probiotics in inflammatory bowel disease: is there a scientific rationale? Inflamm Bowel Dis 2000; 6: 107-115.

Simmering R, Blaut M. Pro- and prebiotics-the tasty guardian angels? Appl Microbiol Biotechnol 2001; 55: 19-28.

Sloan E. The top ten functional food trends. Food Technology 2000; 54: 33-62.

Steidler L, Hans W, Schotte L, et al. Treatment of murine colitis by Lactococcus lactis secreting Interleukin-10. Science 2000; 289: $1352-5$

Suau A, Bonnet R, Sutren M, et al. Direct analysis of genes encoding 16S rRNA from complex communities reveals many novel molecular species within the human gut. Appl Environ Microbiol 1999; 65: 4799-807.

Suau A, Rochet V, Sghir A, et al. Fusobacterium prausnitzii and related species represent a dominant group within the human faecal flora. Syst Appl Microbiol 2001; 24: 139-45.

Steer T, Carpenter H, Tuohy K, Gibson GR. Perspectives on the role of the human gut microbiota in health and methods of study. Nutr Res Rev 2000; 13: 229-54.

Vaughan EE, Mollet B, de Vos WM. Functionality of probiotics and intestinal lactobacilli: light in the intestinal tract tunnel. Curr Opin Biotechnol 1999; 10: 505-10.

Zamfir M, Callewaert R, Calina Cornea P, Savu L, Vatafu I, De Vuyst L. Purification and characterization of a bacteriocin produced by Lactobacillus acidophilus IBB 801. J App1 Microbiol 1999; 87: 923-31. 


\section{APPENDIX}

Cluster Coordinator: Prof. Tina Mattila-Sandhoim

VIT Biotechnology

P.O. Box 1500

FIN-02044 VTT

Fintand

Phone: $\quad+35894565200$, mobille: +358505527243

Fax: $\quad+35894552103$

E-mail: tiina.mattila-sandholm@vtt.fi

\begin{tabular}{|c|c|}
\hline \multicolumn{2}{|c|}{$\begin{array}{l}\text { EU Commission, Scientific Officer: } \\
\text { Dr. Jügen Lucas } \\
\text { European Commission } \\
\text { Research DG, SDME 8-12 } \\
\text { Rue de la Loi } 200 \\
\text { B-1049 Brussels } \\
\text { Belgium }\end{array}$} \\
\hline $\begin{array}{l}\text { Phone: } \\
\text { Fax: } \\
\text { E-mail: }\end{array}$ & $\begin{array}{l}+3222964152 \\
+3222964322 \\
\text { Jurgen.lucas@cec.eu.int }\end{array}$ \\
\hline \multicolumn{2}{|c|}{$\begin{array}{l}\text { Consumer Platfom: Dr. Lisa Lahteenmäk } \\
\text { VTT Biotechnology } \\
\text { P.O. Box } 1500 \\
\text { FIN-02044 VTT } \\
\text { Finland }\end{array}$} \\
\hline $\begin{array}{l}\text { Phone: } \\
\text { Fax: } \\
\text { E-mail: }\end{array}$ & $\begin{array}{l}\text { +35894565965 } \\
\text { +35894552103 } \\
\text { lisa.lahteenmaki@vtt.fi }\end{array}$ \\
\hline
\end{tabular}

Dr. Annick Mercenier

Département de Microbiologie des Ecosystèmes

Rue du Professeur Calmette, 1

59019 Lille

France

Phone: $\quad+33320871186$

Fax: $\quad+33320871192$

E-mail: annick.mercenier@ibl.fr

\section{Dr. Joël Dore}

INRA

Unité d'Ecologie et Physiologie du Système Digestif

Domaine de Vilvert

78352 Jouy-en-Josas Cedex

France

Phone: $\quad+33134652709$

Fax: $\quad+33134652492$

E-mail: dore@jouy.inra.fr

\section{Industry Platfom: Prof. Charies Daly}

University College Cork

Faculty of Food Science \& Technology

Cork

Ireland

Prof. Fergus Shanahan

National University of Ireland

University College Cork

Department of Microbiology and Medicine

Western Road

Cork

Ireland

Phone: $\quad+353214902843$

$\begin{array}{ll}\text { Phone: } & +35321902007 \\ \text { Fax: } & \quad+35321276398\end{array}$

E-mail: nfbc@ucc.ie

Fax: $\quad+353214276318$

E-mail: ntbc@ucc.ie

Science Platform: Prof. Willem de Vos

Wageningen University, Laboratory of Microbiology

Department of Agrotechnology and Food Sciences

Hesselink van Suchtelenweg 4

PO Box 8033

6703 CT Wageningen

The Netherlands

Phone: $\quad+31317483100$

Fax: $\quad+31317483829$

E-mail: willem.devos@algemeen.micr.wau.nl

Project coordinators:

Dr. Michael Blaut

Deutsches institut fur Ernährungsforschung

Potsdam-Rehbrücke

Gastrointestinale Mikrobiologie

Arthur-Scheunert-Alle 114-116

14558 Bergholz-Rehbracke

Germany

Phone: $\quad+493320088470$

Fax: $\quad+493320088407$

E-mail: blaut@www.dife.de

Dr. Dietrich Knorr

Technische Universitat Berlin

Lebensmittelbiotechnologie und -prozesstechnik

Königin-Luise-Strasse 22

14195 Berlin

Germany

Phone: $\quad+493031471250$

Fax: $\quad+49308327662$

E-mail: dietrich.knorr@tu-berlin.de

\section{Dr. Luc De Vuyst}

Vrije Universiteit Brussel

Research Group of Industrial Microbiology

Fermentation Technology and Downstream Processing

Pleinlaan 2

1050-Brussels

Belgium

Phone: $\quad+3226293245$

Fax: $\quad+3226292720$

E-mail: Idvuyst@vub.ac.be

\section{Dr. Herman Goossens}

Universiteit Antwerpen

Universitair Ziekenhuis

Department of Medical Microbiology

Wilrijkstraat 10

2650 Edegem

Belgium

Phone: $\quad+3238213789$

Fax: $\quad+3238254281$

E-mail: herman.goossens@uza.uia.ac.be

Dr. Glenn Gibson

University of Reading

School of Food Biosciences

Whiteknights

RG6 6AP Reading

United Kingdom

Phone: $\quad+441189357223$

Fax: $\quad+441189357222$

E-mail: $\quad$ g.r.gibson@reading.ac.uk 
Copyright of Microbial Ecology in Health \& Disease is the property of Taylor \& Francis Ltd and its content may not be copied or emailed to multiple sites or posted to a listserv without the copyright holder's express written permission. However, users may print, download, or email articles for individual use. 